\title{
Biological Process Qualifier
}

National Cancer Institute

\section{Source}

National Cancer Institute. Biological Process Qualifier. NCI Thesaurus. Code C45419.

A term that helps define and render a biological process concept unique by further defining or describing a specific aspect of a biological process. 